\title{
PIV measurements on a Blowing Flap
}

\author{
Florence V. Hutcheson ${ }^{1}$ \\ NASA Langley Research Center, Hampton, VA, 23681 \\ and \\ Daniel J. Stead ${ }^{2}$ \\ Lockheed Martin Engineering and Sciences, Hampton, VA, 23681
}

\begin{abstract}
PIV measurements of the flow in the region of a flap side edge are presented for several blowing flap configurations. The test model is a NACA 63-215 Hicks Mod-B main-element airfoil with a half-span Fowler flap. Air is blown from small slots located along the flap side edge on either the top, bottom or side surfaces. The test set up is described and flow measurements for a baseline and three blowing flap configurations are presented. The effects that the flap tip jets have on the structure of the flap side edge flow are discussed for each of the flap configurations tested. The results indicate that blowing air from a slot located along the top surface of the flap greatly weakened the top vortex system and pushed it further off the top surface. Blowing from the bottom flap surface kept the strong side vortex further outboard while blowing from the side surface only strengthened the vortex system or accelerated the merging of the side vortex to the flap top surface. It is concluded that blowing from the top or bottom surfaces of the flap may lead to a reduction of flap side edge noise.
\end{abstract}

\section{Introduction}

During airport approach, when the engines of an aircraft are near idle condition, and the high-lift systems and landing gears are deployed, airframe noise is the dominant noise source. The noise that is generated at the side edge of the flaps has been identified as an important airframe noise component ${ }^{1-5}$ and is a target for noise control.

A number of numerical and experimental studies ${ }^{6-13}$ have been conducted in order to identify and model the noise generation mechanisms at the flap side edge. Flow field measurements ${ }^{8}$ in the flap side edge region of a wing with a half-span flap have revealed the presence of a 2-vortex system (see Figure 1): a small vortex near the flap side edge on the top surface and a stronger side vortex along the lower portion of the flap side edge. As it travels downstream along the flap side edge, the side vortex strengthens and expands. At about mid-chord it begins to spill over the flap top surface and merges with the small top vortex. The instabilities in this vortex system and in the strong shear layer that originates on the bottom edge of the flap create an unsteady pressure field at the flap side edge causing sound to radiate. Brooks et al. ${ }^{13}$ have determined that the dominant flap side edge noise regions are located around mid-chord on the pressure side of the flap edge and around 60-65\% chord on the suction side.

Various flap side-edge noise reduction concepts have been evaluated. CFD calculations ${ }^{14}$ showed that the use of porous side-edge treatment lead to the formation of a significantly weaker side-edge vortex system and the elimination of the bursting of the side edge vortex at large flap deflection angles. Both effects are expected to result in a decrease of the radiated noise. Experimentally, it has been shown that substantial noise reduction could be achieved by applying fencing type devices ${ }^{15-16}$ or continuous moldline technology ${ }^{17}$ to the flap tip. Koop et al. ${ }^{18}$ showed that flap tip winglets, and suction side fences were most effective in reducing flap side-edge noise and that pressure side fences and microtabs (vortex generators) were least effective. In the same study, Koop et al. also demonstrated an active flow control method ${ }^{18-19}$ for noise reduction. Air was blown into the flap side edge vortex system through a series of small round orifices located along the flap suction and pressure side edges between 13 and 35\% chord. The blowing caused the vorticity in the flap side-edge shear layer to be concentrated in small vortices (located further away from the flap surface with increasing blowing momentum), the vorticity field on the

\footnotetext{
${ }^{1}$ Research Engineer, Acoustics Branch, 2 North Dryden Street/MS 461

${ }^{2}$ Aerospace Engineer, Acoustics Branch, 2 North Dryden Street/MS 461
} 
suction side of the flap to be more homogeneous and the interaction between the curved shear layer and the suction side edge to be reduced. As a result, very good noise reduction was achieved between $2 \mathrm{kHz}$ and $5 \mathrm{kHz}$.

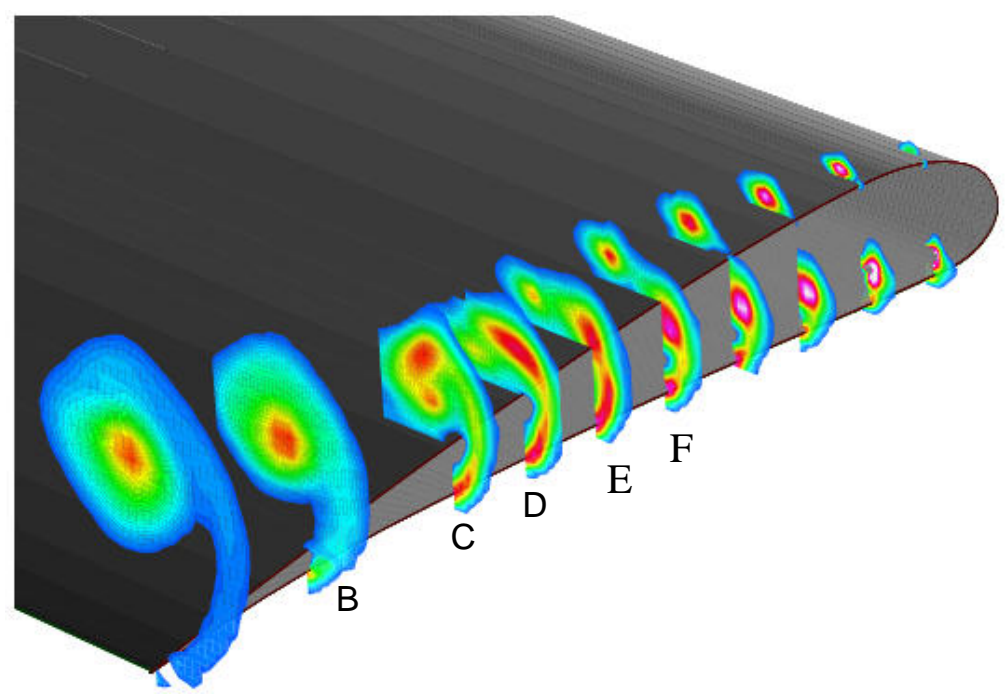

Figure 1. Axial vorticity contours. 5-hole probe measurements (ref. 8). Flap at $29^{\circ}$.

In the present study, the effect of flap tip jets on flap side edge noise is investigated. Air is blown through thin rectangular slots located near or along the flap side edge. Based on the known structure of the flap side edge flow and on the afore mentioned findings by Brooks et al. ${ }^{13}$ regarding the dominant noise source regions, several tip jet configurations were designed. Before acoustic data was acquired, PIV (Particle Image Velocimetry) measurements were performed on a subset of the flap configurations that were designed. These PIV measurements are presented in this paper and the effect of the tip jets on the flap side edge flow field is evaluated.

\section{Test description}

\section{A. Model configuration}

The PIV measurements for the blowing flap test were performed at NASA Langley Research Center in the Quiet Flow Facility (QFF). The QFF is an open jet facility equipped with a 2 by 3 foot rectangular open jet nozzle. The test model is a NACA $63_{2}-215$ Hicks Mod-B main-element airfoil with a half-span Fowler flap. The chord and span of the main airfoil are $40.64 \mathrm{~cm}$ and $91.44 \mathrm{~cm}$, respectively. The flap chord is $12.2 \mathrm{~cm}$. As shown in Figure 2, the model was supported above the nozzle by two vertical side plates that are mounted to the short sides of the nozzle. The main airfoil and half-span flap were positioned at angles of attack of $16^{\circ}$ and $29^{\circ}$, respectively. A strip of serrated tape was placed along the main airfoil leading edge to trip the boundary layer. The mean flow Mach number was 0.17 , yielding a Reynolds number of about 1.5 million based on the chord of the main airfoil. 


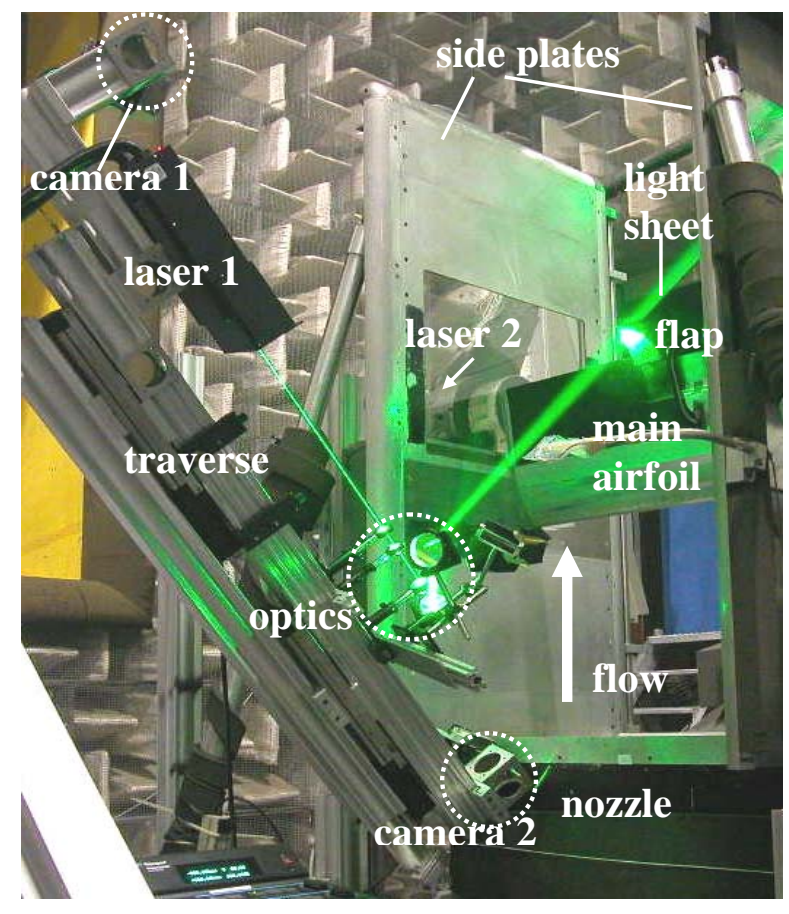

Figure 2. Test set-up

The model used for this test incorporated a partially hollowed flap, with a removable, $6.3 \mathrm{~mm}$ wide, side edge cap (see figure 3). The air blown into the flap exited through small slots located on the side edge cap.

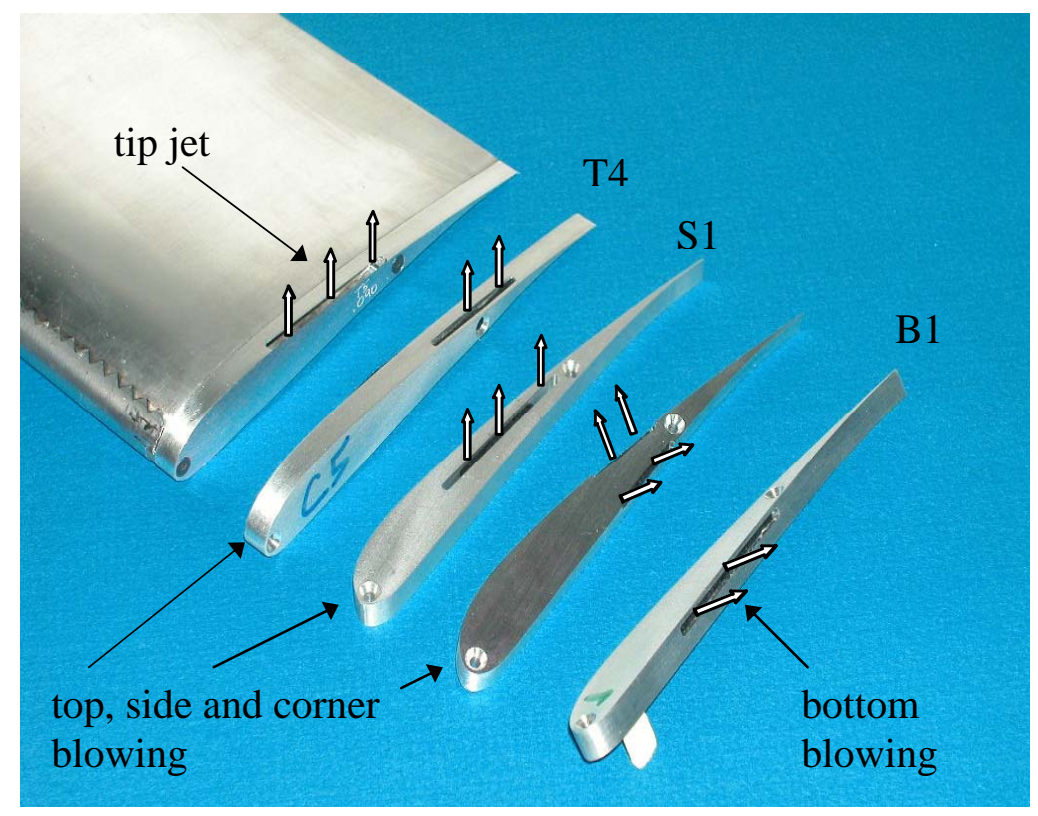

Figure 3. Example of blown flap configurations. 
PIV measurements were performed for four flap side edge configurations. In the first configuration, a solid side edge cap was used, representing the baseline flap. For the other three configurations, air was blown through a thin rectangular slot located on either the top (T4), the side (S1) or the bottom (B1) surface of the flap side edge cap. Configurations T4, S1 and B1 are shown in figure 3. For configuration T4, the slot extended from 50\% chord to $75 \%$ chord (as measured from the flap leading edge), was $2 \mathrm{~mm}$ wide and was located $1 \mathrm{~mm}$ from the flap side edge. For configuration S1, the slot extended from $27 \%$ chord to $60 \%$ chord, was $2 \mathrm{~mm}$ wide and lay $3.8 \mathrm{~mm}$ above the chordline. Finally, for configuration B1, the slot extended along the bottom surface of the flap between 27 and $60 \%$ chord. It was also $2 \mathrm{~mm}$ wide and lay $1 \mathrm{~mm}$ from the flap bottom side edge.

The blowing flap tip jet Mach numbers tested were 0.075, 0.11 and 0.17. A mass flow meter and pressure regulator in the blowing flap air supply line were used to measure and control the flap tip jet speed.

\section{B. PIV system set up}

The flap side edge flow field was measured using stereo PIV. Such PIV configurations yield three components (3-C) of velocity over a two-dimensional plane. The PIV measurements were taken in planes that are parallel to the flap trailing edge and perpendicular to the flap chord (see Figure 4). These measurement planes were respectively located at 42, 51, 59, 67, 83 and $110 \%$ chord (where $0 \%$ and $100 \%$ chord corresponds to the flap leading and trailing edges). Note that 5-hole probe measurements (shown in Figure 1) were also taken at these locations, except for $110 \%$ chord.

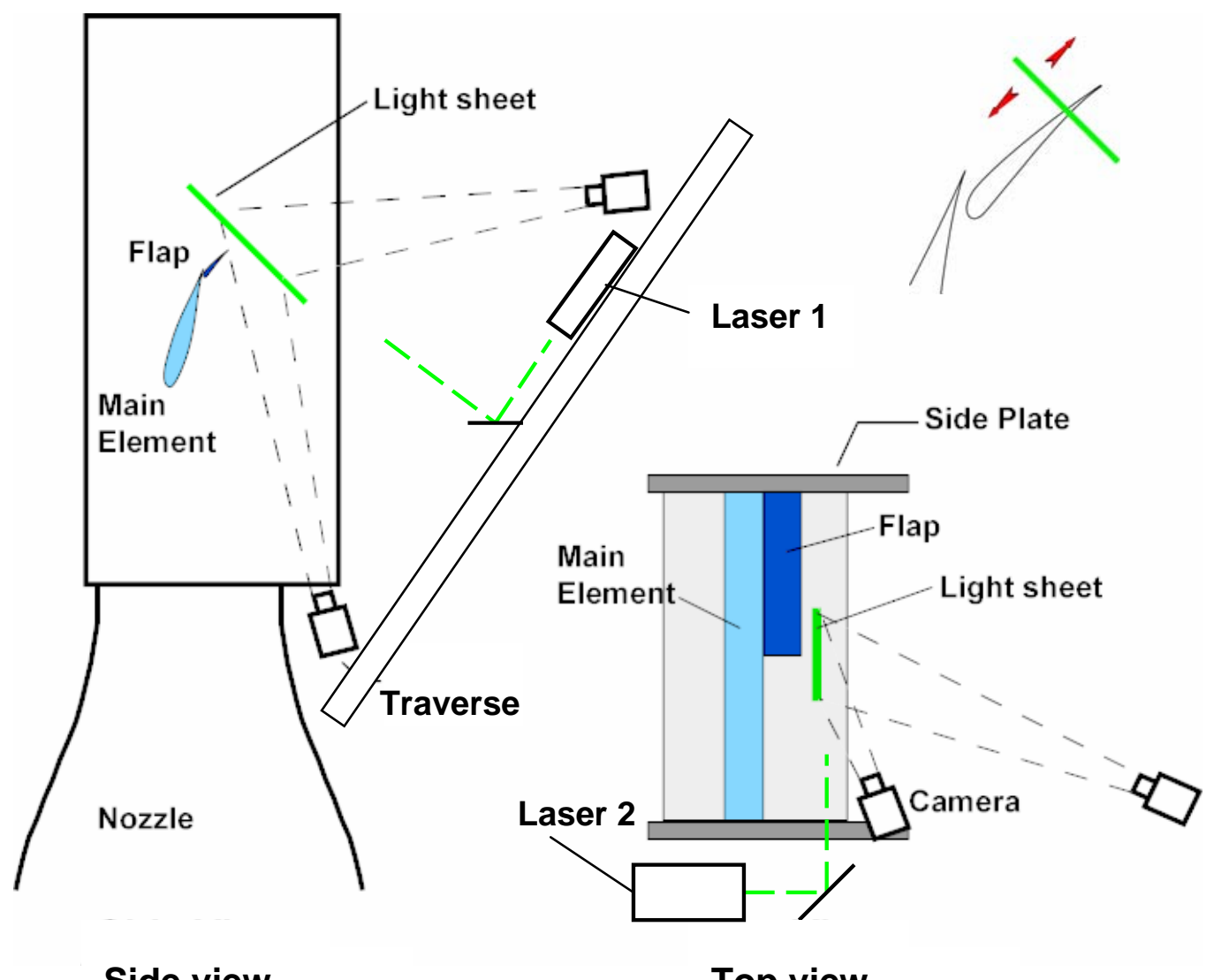

Side view

$\underline{\text { Top view }}$

Figure 4. PIV system set up 
The measurement planes were illuminated using two Nd:YAG double cavity lasers with a repetition rate of 15 $\mathrm{Hz}$. To minimize laser reflections from the flap surfaces, the PIV measurements at each flap side edge chordwise location were taken in 3 stages. First (referring to figures 4 and 5), using laser 1, measurements were acquired in the region to the side of the flap edge. Then, using laser 2, measurements were acquired in the region above the flap and finally below the flap. Beam-blocks were used to "chop off" the light sheet as close as possible to the flap surfaces.

Attempts were made to acquire measurements directly along the model surfaces by removing the beam-blocks and applying treatments to the model surfaces to minimize reflections from the light sheet. One of the surface treatments tested consisted of a special fluorescent paint that reflected the laser light in a different wavelength which could be filtered from the recorded images by mounting optical filters to the cameras. Unfortunately, the laser intensity required to sufficiently illuminate the seeded flow lead to a very rapid deterioration of the surface treatment. Limited success was also achieved using flat black paint or polished surfaces (to respectively "absorb" or "cleanly reflect" the incoming light). Replacing the aluminum flap side edge cap by a translucent cap (made of Plexiglas) was also considered, but this option was quickly abandoned because of the "glowing" effect that took place when the laser propagated through the cap.
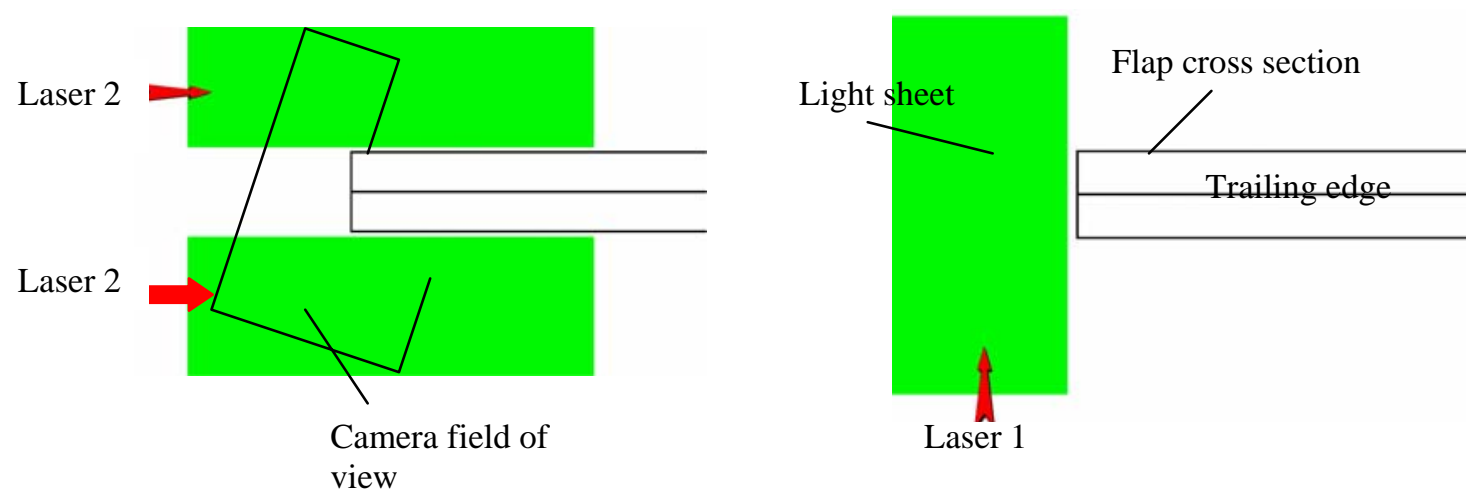

Figure 5. Laser system.

The optical lens system used to produce the light sheets consisted of a series of two spherical lenses (of focal lengths $-200 \mathrm{~mm}$ and $200 \mathrm{~mm}$, respectively) followed by one cylindrical lens. The focal length of the cylindrical lens used with laser 1 was $-40 \mathrm{~mm}$ while the focal length of the cylindrical lens used with laser 2 was $-50 \mathrm{~mm}$. The spacing between the spherical lenses was adjusted such that the thickness of the light sheet was approximately 1 $\mathrm{mm}$. A mirror placed past the cylindrical lens was used to properly orient the light sheet.

Two Redlake MegaPlus ES 1.0 CCD cameras with $105 \mathrm{~mm}$ lenses were used to record the PIV images with a spatial resolution of 1008 by 1012 pixels. Special mounts were designed for the cameras in order to minimize vibration. The lasers, optical lenses and cameras were mounted on a common traverse system in order to keep the distance between the cameras and the light sheet constant while moving to the different measurement locations.

The location of the cameras was limited by the side plates that were supporting the test model and by the jet flow. The best field of view of the flap side edge that could be obtained from the two cameras for a 3-C PIV configuration is sketched in Figures 6. 


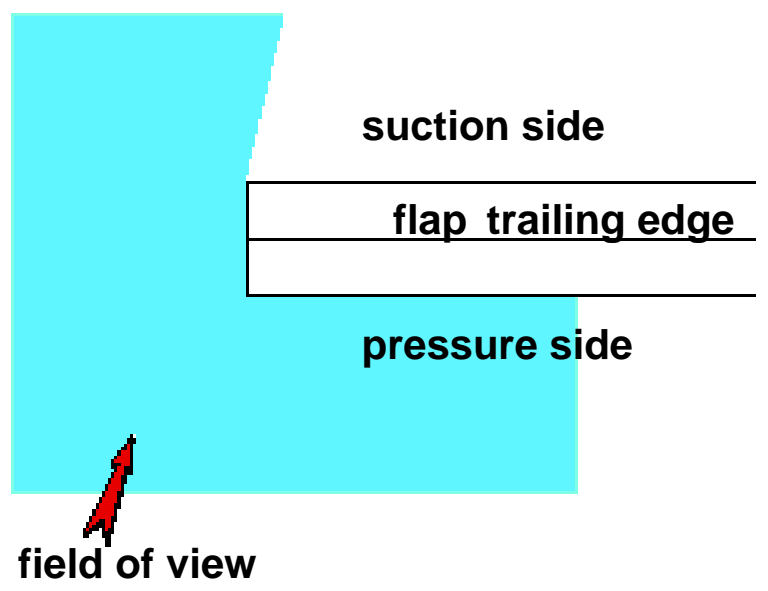

Figure 6. Camera field of view.

The flow was seeded upstream of the low-pressure air fan (i.e., at the beginning of the flow circuit) to ensure a homogeneous distribution of the seeded particles throughout the jet flow. The air that was blown into the flap was also seeded (see figure 7). Laskin nozzle seeders employing with Bis(2-Ethylhexyl) Sebacate were used to generate particles of less than $1 \mu \mathrm{m}$ in diameter.
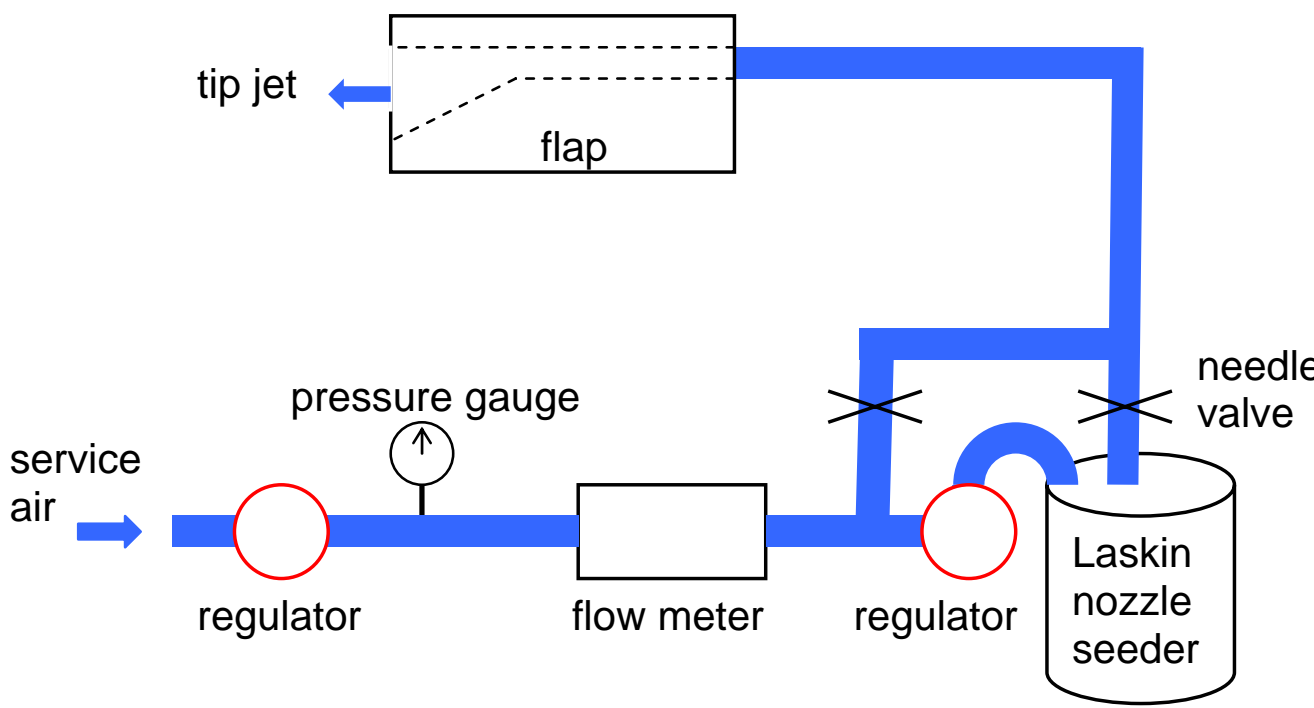

Figure 7. Seeded air injection system.

\section{Data acquisition and processing}

Over 400 image pairs were acquired at each measurement location, at a rate of 15 frames per second (the frame rate was synchronized with that of the laser pulse). Integrated Design Tools Provision software was used to process the PIV images and generate 3-component velocity vectors maps. At each measurement location, the PIV measurements were made over an area of 10 by $8 \mathrm{~cm}$. The PIV images were processed with a 24 by 24 pixel 
interrogation window size with a 50\% overlap, leading to a spatial resolution of 1 velocity vector for each $1.15 \mathrm{~mm}$ by $0.94 \mathrm{~mm}$ area. Cross- correlation techniques ${ }^{20}$ were used to compute the velocity vectors from PIV image pairs. The percentage of interpolated velocity vectors computed in each vector map was kept below $1 \%$ (an interpolated vector is the result of a least square interpolation of nearest neighbors). The computation of interpolated vectors occurred in the portion of the PIV images where the signal to noise ratio was poor because of contamination from reflection, obstruction from the flap model, or low illumination.

\section{Results}

Flow field images obtained from the PIV measurements are presented in this section. They were obtained with the standard data processing methodology described above.

Figures displaying the averaged in-plane velocity field $(u \hat{x}+v \hat{y})$ and averaged axial vorticity field $\left(\Omega_{\mathrm{z}}=\mathrm{dv} / \mathrm{dx}-\mathrm{du} / \mathrm{dy}\right.$ ) are shown for each flap configuration tested. The vectors in each map are only shown for every second node in $\mathrm{x}$ and every fourth node in $\mathrm{y}$. The non-dimensional plane location $\mathrm{z} / \mathrm{c}$ is indicated next to each map where $\mathrm{z}$ is measured from the flap leading edge and along its chord, c. The six measurement planes are labeled as cuts A through F (as in Figure 1, for the 5 hole probe measurements).

\section{A. Baseline flap configuration}

The PIV measurements obtained for the baseline flap are shown in Figure 7. Note that for cut F (the plane nearest the main element airfoil) a zone of inaccurate results exists as shown in the plot. In this region, unwanted laser flare light illuminated the main element producing false correlations and errant velocities.

Comparing Figures 1 and 7, it is seen that the PIV and 5-hole probe measurements agree very well. At 42\% chord (cut F), the vortex that originated along the bottom edge of the flap is creeping up along the side surface and reaching the top edge. At $51 \%$ chord (cut $\mathrm{E}$ ) it is beginning to spill over the top surface while the axial vorticity in the shear layer coming off the bottom edge is strongest. At $59 \%$ chord (cut D), the vortex core has almost fully merged onto the top surface and is centered about $4 \mathrm{~mm}$ above the top surface of the flap. At $67 \%$ chord (cut C), the vortex core is centered about $9 \mathrm{~mm}$ above the top surface. At $83 \%$ chord (cut B), the vortex has a more efficient (round) structure and its core is centered about $12 \mathrm{~mm}$ above the top surface and $2 \mathrm{~mm}$ inboard. These results are consistent with the ones reported in reference 8.

\section{B. Blowing flap configuration T4}

The PIV measurements obtained for the blowing flap configuration T4 are presented in Figures 8 and 9 for a tip jet Mach number of 0.075 and 0.17 , respectively. For this flap configuration the tip jet originates from a slot that extends along the top surface between 50 and $75 \%$ chord. It is seen in these figures that at 59 and $67 \%$ chord where (based on reference 13) the highest levels of the noise radiation takes place, the vortex is greatly weakened, it has been pushed further off the top surface and its structure is deteriorated. These effects are accentuated as the tip jet Mach number increases. At $83 \%$ and $110 \%$ chord (i.e., downstream of where the blowing takes place), the effect of the tip jet on the vortex strength and location can still be seen. The vortex is much weaker than in the baseline case and centered approximately $4 \mathrm{~mm}$ further above the flap. This flap configuration hence leads to a weaker shear flow coming off the flap top side edge. This should result in a reduced level of noise radiating from that edge.

\section{Blowing flap configuration B1}

The PIV measurements obtained for the blowing flap configuration B1, with a tip jet Mach number of 0.17, are presented in Figure 10. For this flap configuration the tip jet extends along the bottom surface from 27 to $60 \%$ chord. The intent of blowing from the bottom surface was to deflect the shear flow that is coming off the bottom edge, i.e., forcing it to go around the edge instead of coming straight off of it. This should reduce the noise radiating from the bottom edge. It can be seen in cuts C, D and E of Figure 10 that the shear layer wrapping around the flap side edge is indeed farther away from the side surface than in the baseline case. The vortex is also not able to move inboard as with the baseline configuration. It is seen however that although the blowing seems to displace the shear layer, it also strengthens it. Compared to the baseline case, cut B reveals a larger, yet elongated vortex core located partially outboard of the flap side edge. The resulting effect on the radiated noise will have to be seen. Similar flow effects were observed when blowing with a tip jet Mach number of 0.11 . Blowing with a tip jet Mach number of 0.075 , however, did not appear to have any significant effects on the flow. 


\section{Blowing flap configuration S1}

The PIV measurements obtained for the blowing flap configuration S1, with a tip jet Mach number of 0.17 , are presented in Figure 11. For this flap configuration the tip jet is located along the side surface between 27 and $60 \%$ chord. The intent of blowing from the side surface was to "build a retaining wall" to slow down the travel of the strong side vortex to the top edge and hence delay its merger onto the top surface and shorten the portion of the flap top surface over which the vortex strong shear layer "rubs" against the top edge (causing noise to radiate). The PIV results seem to indicate that the opposite effect was achieved. It is seen on Figure 11 that compared to the baseline case, the merging of the side vortex to the top surface was accelerated, and the vortex is seen to also "lift off" the flap surface sooner. Thus, the structure of the flow seen in cuts E, D and C of Figure 11 corresponds to that of cuts D, C and B in Figure 7. This was observed for the 0.11 and 0.17 tip jet Mach number cases. For the 0.075 tip jet Mach number case, the blowing only contributed to "feed" the shear layer and strengthen the vortex system. Therefore, for all the tip jet Mach numbers tested, this blowing flap configuration is expected to lead to an increase of the radiated noise level.

\section{Conclusions}

PIV flow measurements for a baseline and three blowing flap configurations were presented. The results indicate that reduction of the noise radiating from the flap side edge is more likely to be achieved by blowing air from a slot located along the top surface of the flap (configuration T4). It was shown that the blowing greatly weakened the top vortex system and pushed it further off the top surface. These beneficial effects occurred with the lowest tip jet Mach number tested and were accentuated at the higher tip jet speeds.

Blowing from the bottom flap surface was found to strengthen but also to deflect and push the shear layer away from the flap edge, keeping the strong side edge vortex further outboard. This was observed only for the two highest tip jet Mach numbers tested. With a sufficiently high enough tip jet speed, this flap configuration may therefore also lead to noise reduction.

Finally, the results indicated that for the tip jet speeds tested, blowing from the side surface only strengthens the vortex system or accelerates the merging of the side vortex to the top surface. This blowing flap configuration is therefore likely to lead to noise increase. 


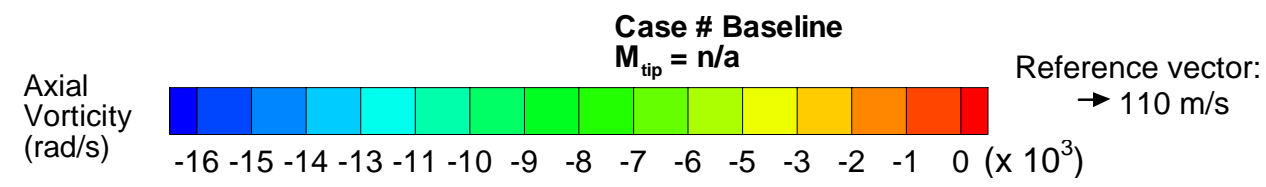

Cut A

$\mathrm{Z} / \mathrm{c}=1.1$
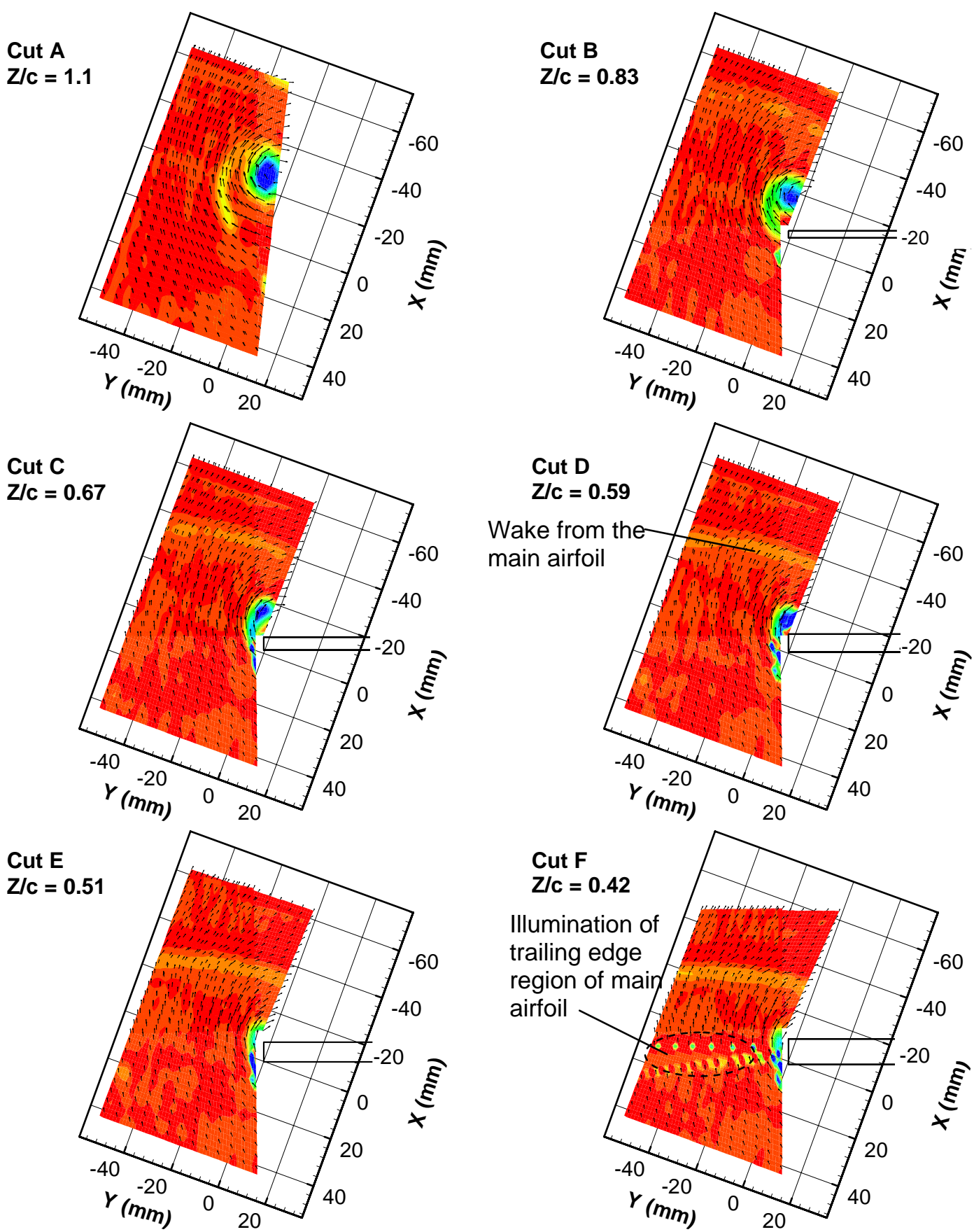

Figure 7. Baseline configuration. Axial vorticity contours and in-plane velocity vectors. 

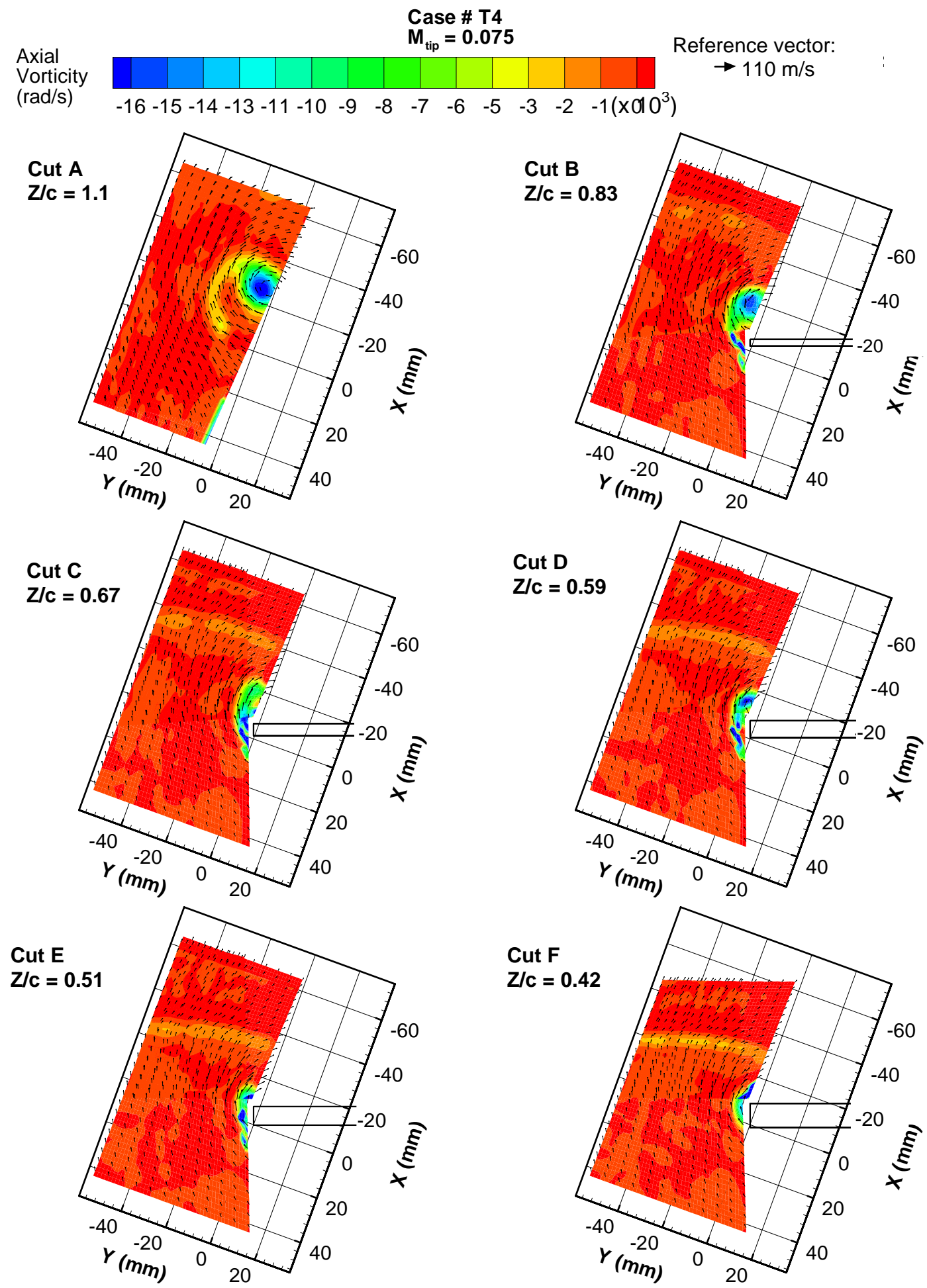

Figure 8. Configuration T4. Tip jet Mach number is 0.075 . 


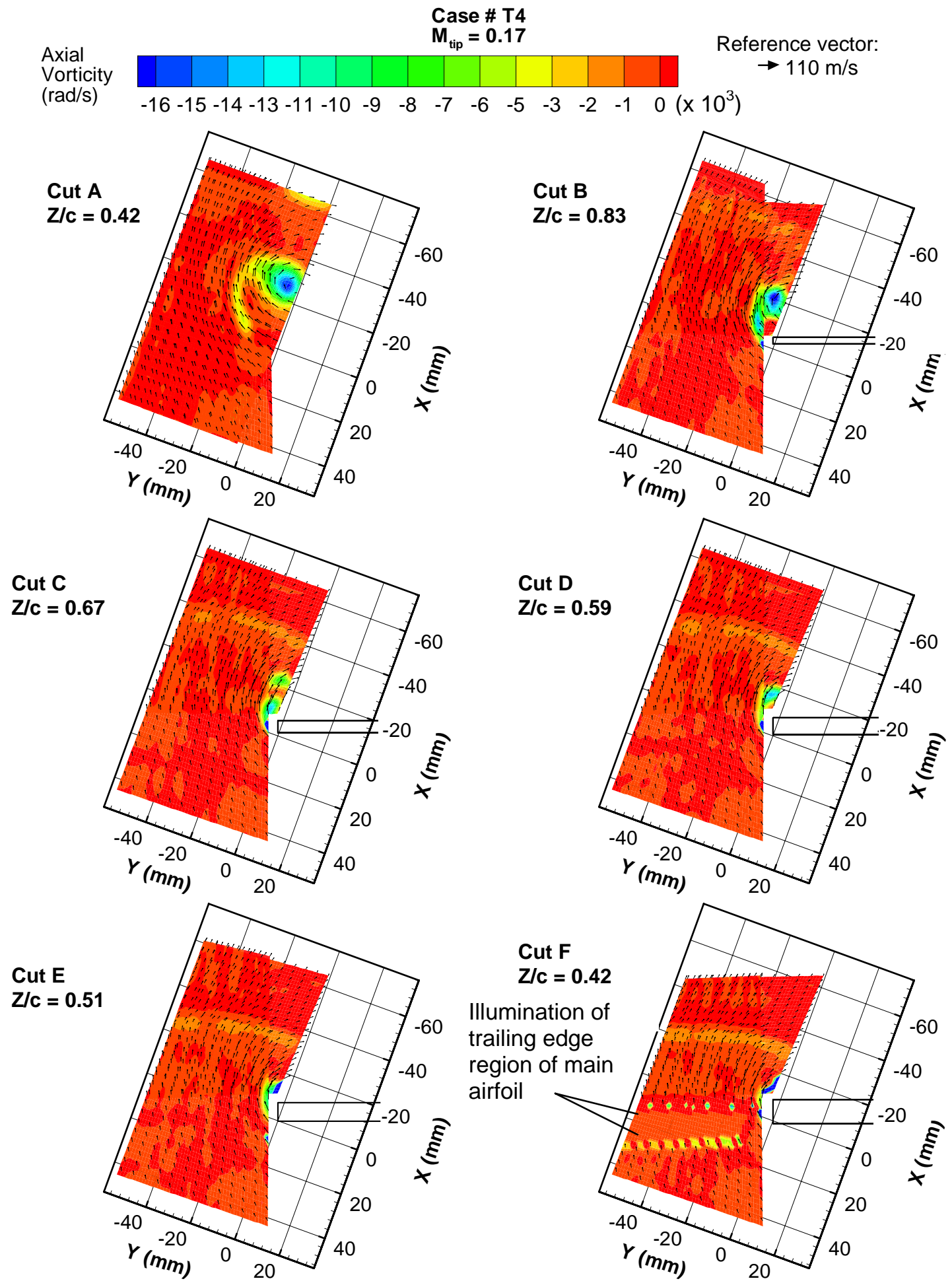

Figure 9. Configuration T4. Tip jet Mach number is 0.17. 

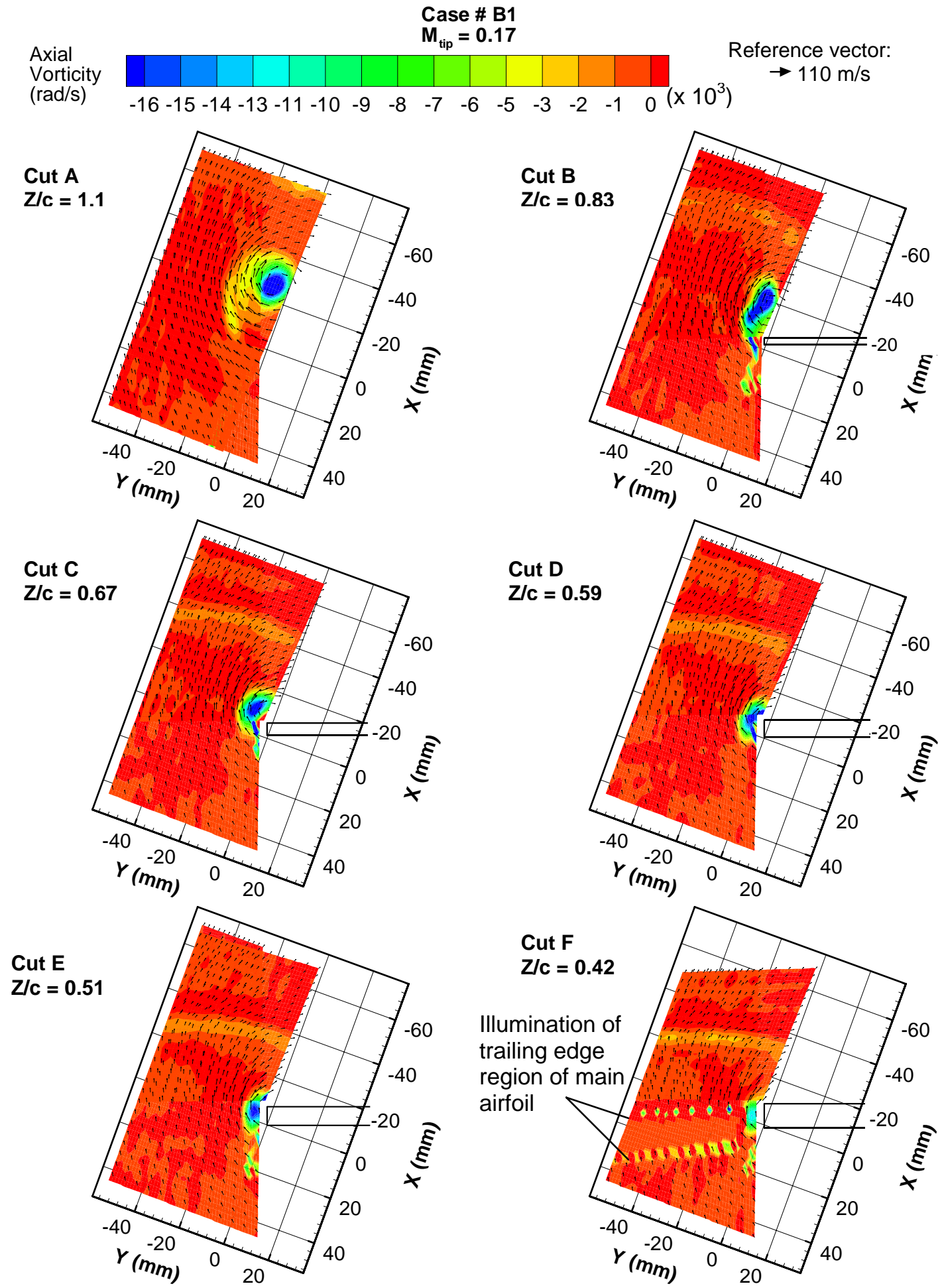

Figure 10. Configuration B1. Tip jet Mach number is 0.17. 

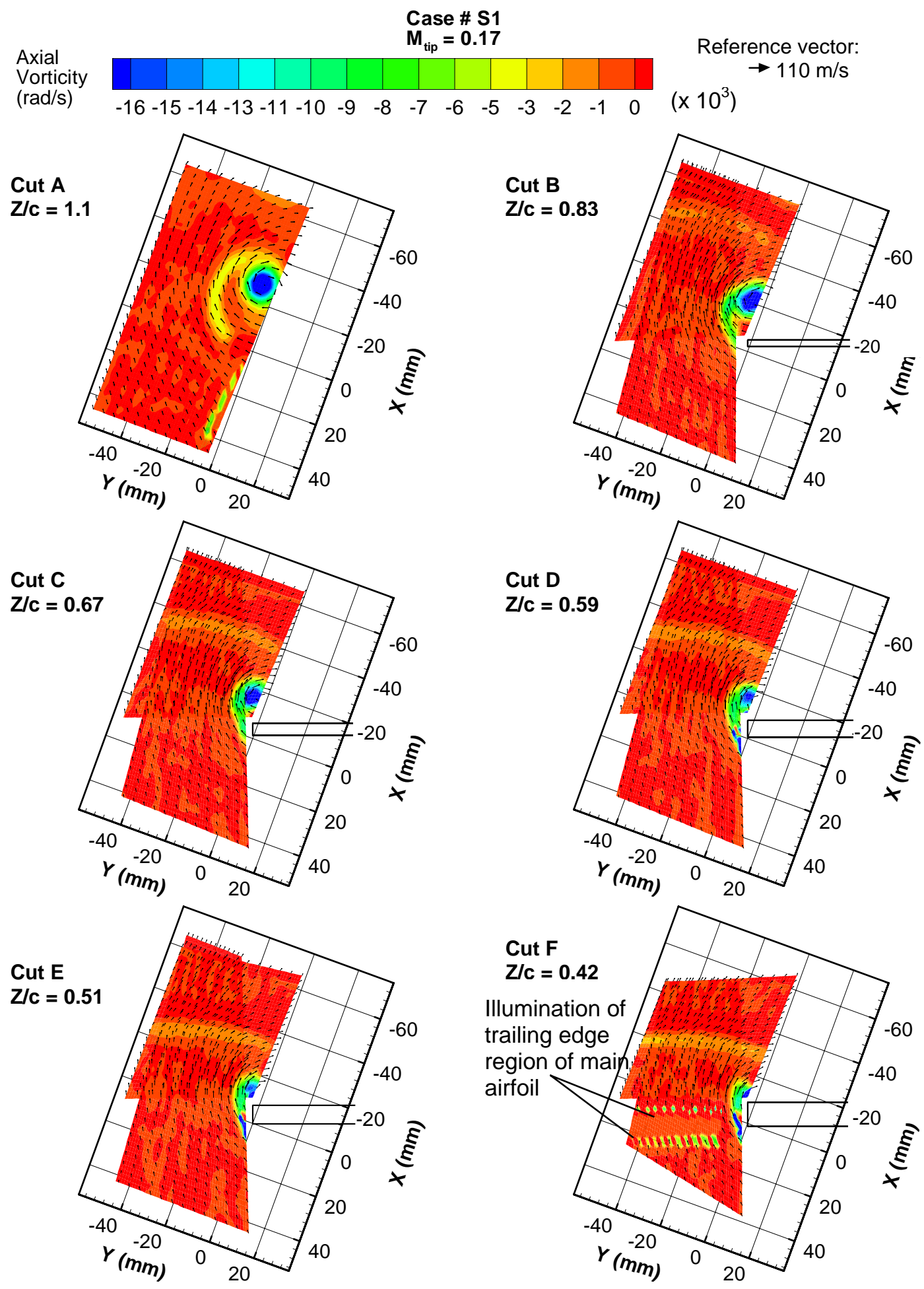

Figure 11. Configuration S1. Tip jet Mach number is 0.17. 


\section{References}

${ }^{1}$ Kendall, J. M.: Measurements of Noise Produced by Flow Past Lifting Surfaces, AIAA paper 78-238, 1978.

${ }^{2}$ Block, P. J.: Assessment of Airframe Noise, Journal of Aircraft, Vol. 16, no. 12, pp. 834-841, Dec. 1979.

${ }^{3}$ Athye, W. F., Miller, W. R. and Meecham, W. C.: Wing and Flap Noise Measured by Near and Far Field Cross Correlation Techniques, AIAA Paper 79-067, March 1979.

${ }^{4}$ Fink, M. R. and Schlinker, R. H.: Airframe Noise Component Interaction Studies, Journal of Aircraft, Vol. 17, no. 2, pp. 99-105, Feb. 1980.

${ }^{5}$ Kendall, J. M. and Athye, W. F.: Noise Generation by a Lifting Wing/Flap Combination at Reynolds Numbers to $2.8 \times 10^{6}$, AIAA Paper 80-0035, 1980.

${ }^{6}$ Khorrami, M. R., Singer, B. A. and Takallu, M. A.: Analysis of Flap Side Edge Flowfield for Identification and Modeling of Possible Noise Sources, SAE Paper 971917, May 1997.

${ }^{7}$ Meadows, K. R., Brooks, T. F., Humpfreys, W. M., Hunter, W. W. and Gerhold, C. H.: Aeroacoustic Measurements of a Wing-Flap Configuration, AIAA Paper 97-1595, May 1997.

${ }^{8}$ Radezrsky, R. H., Singer, B. A. and Khorrami, M. R.: Detailed Measurements of a Flap Side-Edge Flow Field, AIAA Paper 98-0700, 1998.

${ }^{9}$ Khorrami, M. R., Singer, B. A. and Radezrsky, R. H.: Reynolds-Averaged Navier-Stokes Computations of a Flap Side-Edge Flowfield, AIAA Paper 98-0768, 1998.

${ }^{10}$ Takallu, M. A. and Laflin, K. R.: Reynolds-Averaged Navier-Stokes of Two Partial-Span Flap Wing Experiments, AIAA Paper 98-0701, 1998.

${ }^{11}$ Streett, C. L.: Numerical Simulation of Fluctuations Leading to Noise in a Flap-edge Flowfield, AIAA Paper 98-0628, 1998.

${ }^{12}$ Guo, Y. P.: Prediction of Flap Side Edge Noise, AIAA Paper 99-1804, 1999.

${ }^{13}$ Brooks, T. F. and Humphreys, W. M: Flap Edge Aeroacoustic Measurements and Predictions, AIAA Paper A00-31126.

${ }^{14}$ Choudhari, M. and Khorrami, M.: Computational Study of Porous Treatment for Altering Flap Side-Edge Flow Field, AIAA Paper 2003-3113.

${ }^{15}$ Slooff, J. W., de Wolf, W. B., van der Wal, H. M. and Maseland, J. E.: Aerodynamic and Aero-acoustic Effects of Flap Tip Fences, AIAA Paper 2002-0848.

${ }^{16}$ van der Wal, H. and Sijtsma, P.: Flap Noise Measurements in a Closed Wind Tunnel with a Phased Array, AIAA Paper 2001-2170.

${ }^{17}$ Storms, B., Hayes, J., Jaeger, S. and Soderman, P.: Aeroacoustic Study of Flap-Tip Noise Reduction Using Continuous Moldline Technology, AIAA Paper 2000-1976.

${ }^{18}$ Koop, L. and Dillmann, A.: Reduction of Flap Side Edge Noise Passive and Active Flow Control, AIAA 2004-2803.

${ }^{19}$ Koop, L., Ehrenfried, K. and Dillmann, A.: Reduction of Flap Side Edge Noise by Active Flow Control, AIAA $2002-2469$.

${ }^{20}$ Raffel, M., Willert, C. and Kompenhans, J.: Particle Image Velocimetry, a Practical Guide, Springer-Verlag Berlin Heidelberg New York, 1998. 